T Barbui ${ }^{1,2}$, A Carobbio ${ }^{1}$, G Finazzi ${ }^{2}$, P Guglielmelli ${ }^{3}$, S Salmoiraghi ${ }^{2}$ V Rosti ${ }^{4}$, A Rambaldi ${ }^{2}$, AM Vannucchi ${ }^{3}$ and G Barosi ${ }^{4}$

${ }^{1}$ Fondazione per la Ricerca Ospedale Maggiore (FROM), Ospedale Papa Giovanni XXIII, Bergamo, Italy; ${ }^{2}$ Divisione di Ematologia, Ospedale Papa Giovanni XXIII, Bergamo, Italy;

${ }^{3}$ Dipartimento di Medicina Sperimentale e Clinica, Università degli studi di Firenze, Florence, Italy and

${ }^{4}$ Laboratorio di Epidemiologia Clinica e Centro per lo Studio della Mielofibrosi, Fondazione IRCCS Policlinico S Matteo, Pavia, Italy

E-mail: tbarbui@hpg23.it

\section{REFERENCES}

1 Vainchenker W, Favale F. Myelofibrosis, JAK2 inhibitors and erythropoiesis. Leukemia 2013; 27: 1219-1223.

2 Vannucchi AM, Lasho TL, Guglielmelli P, Biamonte F, Pardanani A, Pereira A et al. Mutations and prognosis in primary myelofibrosis. Leukemia 2013; 27: 1861-1869.

3 Cervantes F, Dupriez B, Passamonti F, Vannucchi AM, Morra E, Reilly JT et al. Improving survival trends in primary myelofibrosis: an international study. J Clin Oncol 2012; 30: 2981-2987.

4 Tefferi A. Primary myelofibrosis: 2013 update on diagnosis, risk-stratification, and management. Am J Hematol. 2013; 88: 141-150.

5 Tefferi A, Lasho TL, Huang J, Finke C, Mesa RA, Li CY et al. Low JAK2V617F allele burden in primary myelofibrosis, compared to either a higher allele burden or unmutated status, is associated with inferior overall and leukemia-free survival. Leukemia 2008; 22: 756-761.

6 Poletto V, Rosti V, Villani L, Catarsi P, Carolei A, Campanelli R et al. A3669G polymorphism of glucocorticoid receptor is a susceptibility allele for primary myelofibrosis and contributes to phenotypic diversity and blast transformation Blood 2012; 120: 3112-3117.

7 Tefferi A, Vaidya R, Caramazza D, Finke C, Lasho T, Pardanani A. Circulating interleukin (IL)-8, IL-2R, IL-12, and IL-15 levels are independently prognostic in primary myelofibrosis: a comprehensive cytokine profiling study. J Clin Oncol 2011; 29: 1356-1363.

8 Verstovsek S, Mesa RA, Gotlib J, Levy RS, Gupta V, DiPersio JF et al. A double-blind, placebo-controlled trial of ruxolitinib for myelofibrosis. N Engl J Med 2012; 366 : 799-807.

9 Barbui T, Carobbio A, Finazzi G, Vannucchi AM, Barosi G, Antonioli E et al. Inflammation and thrombosis in essential thrombocythemia and polycythemia vera: different role of C-reactive protein and pentraxin 3. Haematologica 2011; 96: 315-318.

10 Vardiman JW, Thiele J, Arber DA, Brunning RD, Borowitz MJ, Porwit A et al. The 2008 revision of the World Health Organization (WHO) classification of myeloid neoplasms and acute leukemia: rationale and important changes. Blood 2009; 114: 937-951.

11 Barosi G, Mesa RA, Thiele J, Cervantes F, Campbell PJ, Verstovsek S et al. Proposed criteria for the diagnosis of post-polycythemia vera and post-essential thrombocythemia myelofibrosis: a consensus statement from the international working group for myelofibrosis research and treatment. Leukemia 2008; 22: 437-438.

12 Harrell Jr FE, Lee KL, Mark DB. Multivariable prognostic models: issues in developing models, evaluating assumptions and adequacy, and measuring and reducing errors. Stat Med 1996; 15: 361-387.

13 Tefferi A, Pardanani A, Gangat N, Begna KH, Hanson CA, Van Dyke DL et al. Leukemia risk models in primary myelofibrosis: an International Working Group study. Leukemia 2012; 26: 1439-1441.

14 Hasselbalch HC. Chronic inflammation as a promotor of mutagenesis in essential thrombocythemia, polycythemia vera and myelofibrosis. A human inflammation model for cancer development? Leuk Res 2013; 37: 214-220.

Supplementary Information accompanies this paper on the Leukemia website (http://www.nature.com/leu)

OPEN

\title{
The CIBMTR score predicts survival of AML patients undergoing allogeneic transplantation with active disease after a myeloablative or reduced intensity conditioning: a retrospective analysis of the Gruppo Italiano Trapianto Di Midollo Osseo
}

\section{Leukemia (2013) 27, 2086-2091; doi:10.1038/leu.2013.208}

The prognosis of acute myeloid leukemia (AML) patients undergoing allogeneic hematopoietic stem cell transplantation (HSCT) not in complete remission is poor, ${ }^{1-3}$ although this treatment option remains the only possible curative approach for these patients. ${ }^{4} \mathrm{~A}$ retrospective analysis recently published by European Group for Blood and Marrow Transplantation (EBMT) on primary refractory AML allotransplanted with unrelated donors showed that factors associated with improved survival were the following: having received fewer than three courses of induction therapy, the presence of a lower percentage of bone marrow blast infiltration at transplant and patient cytomegalovirus seropositivity. This allowed the development of a scoring system that identified four groups with survival rates ranging between 44 and $0 \% .^{5}$ However, the largest retrospective analysis on AML patients with active disease at the time of conditioning (1673 patients) has been conducted by the Center for International Blood and Marrow Transplant Research (CIBMTR), which, on five pretransplantation variables (duration of first complete remission (CR) $<6$ months, circulating blasts, donor other than HLA-identical sibling, Karnofsky score less than 90 and poor-risk cytogenetics), also set up a pre-HSCT score defining a 3 -year overall survival (OS) ranging from 42 to $6 \% .{ }^{6}$ Here we report outcome data obtained in Italy in a similar cohort of AML patients (523 patients) allotransplanted with active disease. The primary aim of the study was to externally validate the CIBMTR score in a multicenter, retrospective study setting, evaluating the prognostic power of the score in a wider patient population that included not only those receiving a myeloablative conditioning (MAC) but also those treated with a reduced-intensity conditioning $(\mathrm{RIC})^{7,8}$ (as detailed in Supplementary Table 1) and those grafted with a cord blood. Twenty Italian centers belonging to the Gruppo Italiano Trapianto di Midollo Osseo (GITMO) participated in this retrospective observational study. Data were retrieved from the GITMO database, and missing data or specific queries were asked to each center. Overall, 523 patients (no one enrolled into prospective trials) from 20 GITMO centers were included in this 
Table 1. Patients characteristics with univariate and multivariate analysis of prognostic factors for survival

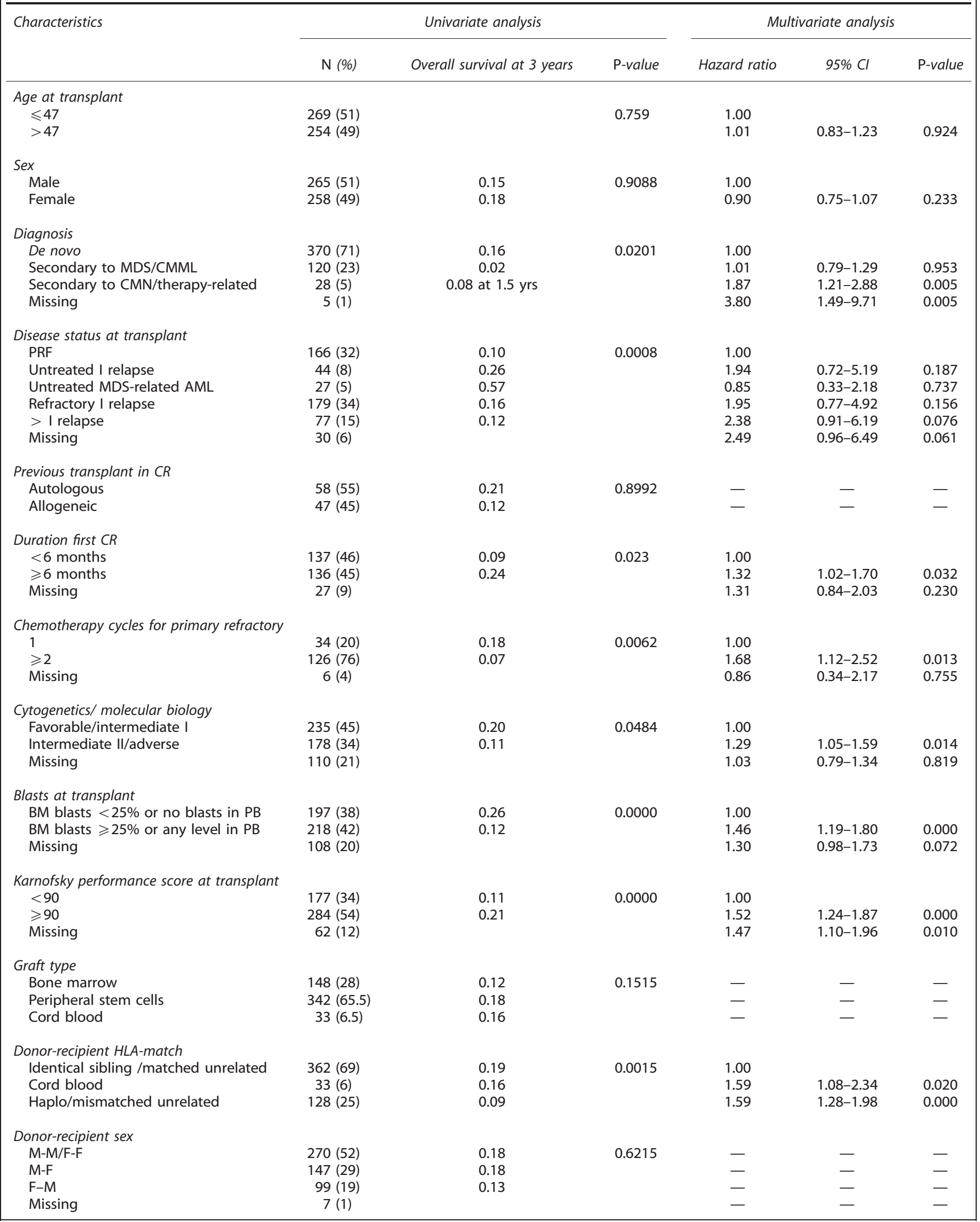


Table 1. (Continued)

\begin{tabular}{|c|c|c|c|c|c|c|}
\hline \multirow[t]{2}{*}{ Characteristics } & \multicolumn{3}{|c|}{ Univariate analysis } & \multicolumn{3}{|c|}{ Multivariate analysis } \\
\hline & $\mathrm{N}(\%)$ & Overall survival at 3 years & P-value & Hazard ratio & $95 \% \mathrm{Cl}$ & P-value \\
\hline \multicolumn{7}{|l|}{ Donor anti-CMV antibodies } \\
\hline Positive & $312(60)$ & 0.17 & 0.8963 & 1.00 & & \\
\hline Negative & $160(31)$ & 0.18 & & 1.03 & $0.84-1.26$ & 0.786 \\
\hline Missing & $51(9)$ & & & 1.35 & $0.81-2.24$ & 0.248 \\
\hline \multicolumn{7}{|l|}{ Patient anti-CMV antibodies } \\
\hline Positive & $421(80)$ & 0.18 & 0.1908 & 1.00 & & \\
\hline Negative & $61(12)$ & 0.17 & & 1.42 & $1.07-1.88$ & 0.015 \\
\hline Missing & $41(8)$ & & & 0.81 & $0.45-1.45$ & 0.473 \\
\hline \multicolumn{7}{|l|}{ Conditioning regimen } \\
\hline RIC & $191(37)$ & 0.16 & 0.9511 & 1.00 & & \\
\hline MAC & $324(62)$ & 0.17 & & 0.96 & $0.79-1.17$ & 0.690 \\
\hline Missing & $8(2)$ & & & 1.12 & $0.50-2.53$ & 0.780 \\
\hline \multicolumn{7}{|l|}{ Type of conditioning } \\
\hline Busulfan/TBI > 600 Gy & $288(55)$ & 0.13 & 0.1614 & - & - & - \\
\hline Others & $219(42)$ & 0.19 & & - & - & - \\
\hline Missing & $16(3)$ & & & - & - & - \\
\hline \multicolumn{7}{|l|}{ GVHD prophylaxis } \\
\hline Ex vivo T-cell depletion & $42(8)$ & 0.05 & 0.0370 & - & - & - \\
\hline (Tacrolimus or CsA) + MTX \pm other & 307 (59) & 0.18 & & - & - & - \\
\hline (Tacrolimus or CsA) \pm other & 77 (15) & 0.17 & & - & - & - \\
\hline Other & $50(10)$ & 0.25 & & - & - & - \\
\hline Missing & $47(9)$ & & & - & - & - \\
\hline \multicolumn{7}{|l|}{$T$-cell depletion in vivo } \\
\hline No T-cell depletion in vivo & $249(48)$ & 0.19 & 0.1220 & - & - & - \\
\hline ATG/ALG/Campath & $196(37)$ & 0.17 & & - & - & - \\
\hline Missing & $78(15)$ & & & - & - & - \\
\hline \multicolumn{7}{|l|}{ Acute GVHD } \\
\hline No & $273(52)$ & 0.15 & & - & - & - \\
\hline Yes & $228(44)$ & & & - & - & - \\
\hline Grade 1 & $91(40)$ & 0.21 & 0.0000 & - & - & - \\
\hline Grade 2 & $71(31)$ & 0.28 & & - & - & - \\
\hline Grade 3 & $41(18)$ & 0.07 & & - & - & - \\
\hline Grade 4 & $25(11)$ & 0.04 & & - & - & - \\
\hline Missing & $22(4)$ & & & - & - & - \\
\hline \multicolumn{7}{|l|}{ Chronic GVHD } \\
\hline No & $286(55)$ & 0.10 & 0.0000 & - & - & - \\
\hline Yes & $126(24)$ & 0.39 & & - & - & - \\
\hline Missing & $111(21)$ & & & & & \\
\hline
\end{tabular}

Abbreviations: ALG, anti-lymphocyte-globuline; AML, acute myeloid leukemia; ATG, anti-tymocyte-globuline; BM, bone marrow; CMML, chronic myelomonocytic leukemia; CMN, chronic myeloproliferative neoplasm; CMV, cytomegalovirus; CR, complete remission; CsA, cyclosporine; GVHD, graft versus host disease; MAC, myeloablative conditioning; MDS, myelodysplastic syndrome; MTX, methotrexate; PB, peripheral blood; PRF, primary refractory; RIC, reduced-intensity conditioning; TBI, total body irradiation.

study. Patient, disease and transplant characteristics are listed in Table 1. The median age was 47.6 (range 18-72). At time of conditioning, AML was defined as primary refractory (patients not achieving a CR after the first induction chemotherapy), MDSrelated (untreated patients with $>20 \%$ bone marrow blasts), untreated first relapse (patients not receiving a salvage chemotherapy before conditioning), refractory first relapse (patients not achieving a remission after a salvage chemotherapy), second or further relapse (untreated or refractory to further salvage chemotherapy).

A marrow blast infiltration $>25 \%$ or any level of peripheral blood (PB) blasts was found in $42 \%$. Donors were HLA identical sibling or matched unrelated in $69 \%$, a family or unrelated mismatched in $25 \%$ and a cord blood unit in $6 \%$. More than $60 \%$ of patients received a MAC and $37 \%$ received a
RIC program. A T-cell depletion was performed in vivo in $37 \%$ and ex vivo in $8 \%$ of patients as described elsewhere. . $^{-11}$

Neutrophil and platelet engraftment was achieved in $87 \%$ of patients after a median of 17 (9-63) and 18 (2-117) days, respectively. Acute graft versus host disease (GVHD) was registered in $46 \%$ of patients (grade $\geqslant 2$ in $60 \%$ of cases), whereas chronic GVHD occurred in $31 \%$ (judged as extended in half of cases). The 1-year cumulative incidence of acute GVHD was $39 \%$, being $28 \%$ for grade $1-2$ and $11 \%$ for grade $3-4$, whereas that of chronic GVHD was $20 \%$. In all, 75 patients (14\%) died early, within 45 days from allotransplant, 282 patients (54\%) achieved CR after allotransplant. Of these latter patients 155 (55\%) relapsed after a median time of 3.7 months (0.4-83). Among the 427 patients who died after HSCT (82\%), 91 were leukemia free. The median followup of the whole patient cohort was 5.3 months (0.10-133), 

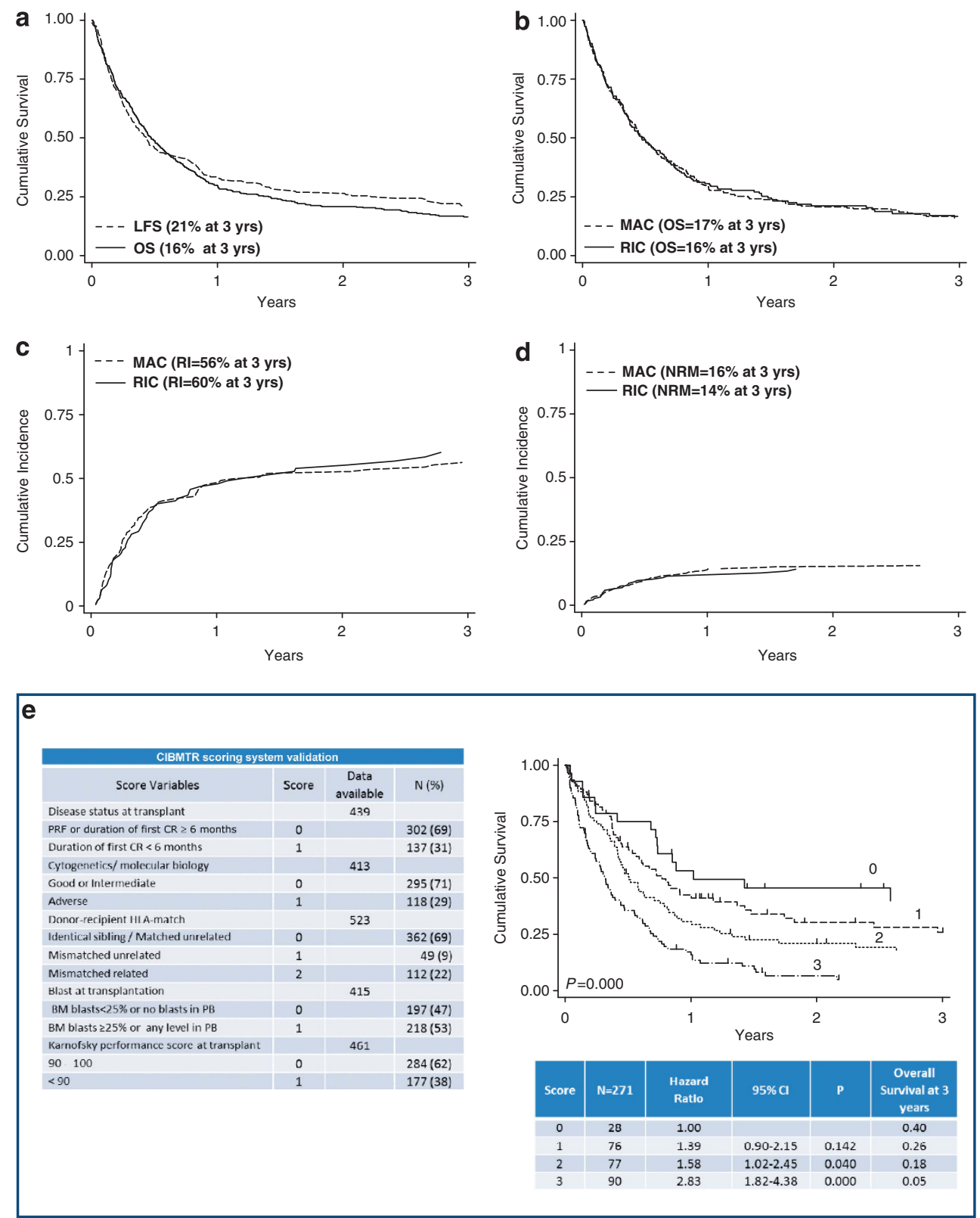

Figure 1. (a) Kaplan-Meier estimates of overall survival, (OS, solid line, $n=523$ ) and leukemia-free survival (LFS, dotted line, $n=282$ ). (b) Kaplan-Meier estimates of overall survival for patients receiving a myeloablative (MAC, dotted line, $n=332$ ) or a reduced-intensity (RIC, solid line, $n=191$ ) conditioning regimen. (c) Incidence of relapse in patients receiving a MAC (dotted line) or RIC (solid line) conditioning regimen. (d) Non-relapse mortality (NRM) in patients receiving a MAC (dotted line) or RIC (solid line) conditioning regimen. (e) A CIBMTR scoring system validation with overall survival according to the risk score. The hazard ratio and the overall survival for score 2 and 3 proved significantly worse than 1 and 2 .

whereas that of survivors was 26 months (1-133) with 96 patients alive and 77 leukemia free. At 3-years, the cumulative incidence of non-relapse mortality (NRM) was $16 \%$. The leukemia-free survival (LFS, calculated from the time of CR after transplantation to death for any cause or relapse) ${ }^{12}$ was $21 \%$, whereas the OS was $16 \%$ (Figure 1).

Eight pre-HSCT variables that negatively influenced survival were identified by univariate and multivariate analysis: an $A M L$ secondary to a previous $C M N$ or a therapy-related $\operatorname{AML}(P=0.005)$, a relapsed $A M L$ with a first $C R$ duration $<6$ months $(P=0.032)$, a primary refractory AML after $\geqslant 2$ chemotherapy cycles pre-HSCT $(P=0.013)$, an intermediate II/adverse cytogenetics $(P=0.014), \mathrm{BM}$ blasts $\geqslant 25 \%$ or any level of PB at HSCT $(P=0.000)$, a Karnofsky performance score $<90 \quad(P=0.000)$, a mismatched related/ unrelated donor $(P=0.020)$ and the presence of patient antiCMV antibodies $(P=0.015)$ (Table 1$)$. To elucidate the impact of the conditioning regimen on main outcomes, the clinical characteristics of patients who received a RIC $(n=191)$ were compared with those of patients receiving a MAC transplant $(n=324)$. A stratified analysis according to the conditioning regimen was developed, and pre-transplantation variables of the two patients groups were compared using the Fisher exact test for categorical variables. Patients receiving a RIC transplant were older $(P=0.000)$, and more frequently were grafted with $\mathrm{PB}$ stem cells $(P=0.000)$ or a mismatched donor $(P=0.002)$ (data not shown). Nonetheless, the intensity of the conditioning regimen did not show an impact on 3-year OS, as well as on the relapse and NRM (Figure 1). 
The OS of our patient cohort was finally analyzed according to the risk categories defined by the CIBMTR score (Table 1). In the more favorable prognostic group of 28 patients (10.5\%) (score 0 ), the OS at 3 years was $40 \%$ (HR 1.00). Similarly to what observed in the original CIBMTR cohort, in the intermediate-I risk group (score 1) $(n=76,28 \%)$ the OS at 3 years was $26 \%$ (HR 1.39, $P=0.142$ ), whereas in the intermediate-II risk group (score 2$)(n=77,28.5 \%)$ and the poor risk group (score 3$)$ ( $n=90$ patients, 33\%) the OS was $18 \%$ (HR 1.58, $P=0.040)$ and $5 \%$ (HR 2.83, $P=0.000)$, respectively (Figure 1 ).

Therefore, the long-term overall- and event- free survival observed in this group of patients are remarkably in keeping with those reported by CIBMTR $^{6}$ and EBMT. ${ }^{5}$ In the GITMO database, the five easy-to-apply pre-HSCT variables defining the CIBMTR score were available contemporarily for only $52 \%$ of the patients analyzed, so that the score could be attributable only to a total of 271 patients. Nonetheless, we can reasonably confirm that the CIBMTR score is an effective and reproducible approach for predicting survival of this group of AML patients at poor prognosis.

However, some important differences between patients analyzed by CIBMTR, EBMT and GITMO must be underlined. First, in the CIBMTR study, only patients who received a total body irradiation or busulfan-based MAC regimen were analyzed, whereas patients receiving a Fludarabine-based or any other RIC regimen were excluded. In the GITMO cohort, a RIC was given to $37 \%$ of patients. In addition, we included also patients receiving a cord blood transplant (6\%), as well as patients with an untreated, MDS-related AML (5\%). In the EBMT experience, patients were limited only to those with a primary refractory AML and those who received an unrelated donor transplant. Despite these differences, our results confirm the EBMT analysis as to the negative impact of a heavy leukemic bone marrow infiltration and the role of the total number of chemotherapy cycles before the conditioning regimen. The prognostic role played by CMV was also underlined in both analysis, although the GITMO results point out the negative impact of a positive serology of the patient while the EBMT suggests that of the negative patient serology. In this study, patients with an AML secondary to a previous CMN or therapy-related had a remarkably poor outcome, and this turned out to be a novel significant adverse prognostic factor However, the poor outcome of AML developing in patients with a previous history of chronic myeloproliferative disorders is not surprising. $^{13}$

Importantly, by univariate and multivariate analysis, the conditioning intensity did not have an impact on 3-year OS and LFS on the entire GITMO cohort. Although the retrospective nature of the study suggests caution, this result may represent a new finding and it is tempting to speculate that for chemo-resistant disease the MAC may not be effective anyhow, so that only patients with an active graft versus leukemia reaction may actually benefit from the transplant.

In conclusion, we have validated the CIBMTR prognostic score in this relatively large patient population with active $A M L$ at allotransplant. It may therefore be possible to identify the patients with advanced $A M L$, who may benefit more from an allogeneic transplant, and this may be relevant for patient counseling. The fact that RIC regimens could also be effective is encouraging for the older patient population, who may be eligible for this procedure.

\section{CONFLICT OF INTEREST}

The authors declare no conflict of interest.

\section{ACKNOWLEDGEMENTS}

This work was supported in part by grants from Associazione Italiana per la Ricerca contro il Cancro (AIRC) and Associazione Italiana Lotta alla Leucemia (AIL).
E Todisco ${ }^{1}$, F Ciceri $^{2}$, E Oldani ${ }^{3}$, C Boschini ${ }^{3}$, C Micò $^{3}$, MT VanLint $^{4}$, I Donnini ${ }^{5}$, F Patriarca ${ }^{6}$, PE Alessandrino ${ }^{7}, \mathrm{~F} \mathrm{Bonifazi}^{8}$, W Arcese ${ }^{9}$, W Barberi ${ }^{10}$, P Marenco ${ }^{11}$, E Terruzzi $^{12}$, S Cortelazzo ${ }^{13^{\prime}}$ S Santarone ${ }^{14}$, A Proia ${ }^{15}$, P Corradini $^{16}$, E Tagliaferri ${ }^{17}$, S Falcioni ${ }^{18}$ G Irrera $^{19}$, L Dallanegra ${ }^{20}$, L Castagna ${ }^{1}$, A Santoro ${ }^{1}$, A Camboni ${ }^{21}$, N Sacchi ${ }^{22}$, A Bosi $^{5}$, A Bacigalupo $^{4}$ and A Rambaldi ${ }^{3}$

${ }^{1}$ Hematology and Bone Marrow Transplant Units of Humanitas Cancer Center, Rozzano, Italy;

${ }^{2}$ Ospedale San Raffaele, Milano, Italy;

${ }^{3}$ Azienda Ospedaliera Papa Giovanni XXIII, Bergamo, Italy;

${ }^{4}$ Ospedale San Martino, Genova, Italy;

${ }^{5}$ Azienda Ospedaliera di Careggi e Università di Firenze,

Florence, Italy;

${ }^{6}$ Azienda Ospedaliera, Università di Udine, Udine, Italy; ${ }^{7}$ Policlinico San Matteo e Università di Pavia, Pavia, Italy;

${ }^{8}$ Policlinico Sant'Orsola, Università di Bologna, Bologna, Italy;

${ }^{9}$ Policlinico Università di Tor Vergata Roma, Rome, Italy;

${ }^{10}$ Policlinico Università La Sapienza Roma, Rome, Italy;

${ }^{11}$ Ospedale Niguarda Ca' Granda, Milano, Italy;

${ }^{12}$ Ospedale San Gerardo, Monza, Italy;

${ }^{13}$ Ospedale Centrale Regionale, Bolzano, Italy;

${ }^{14}$ Ospedale Spirito Santo, Pescara, Italy;

${ }^{15}$ Azienda Ospedaliera San Camillo-Forlanini, Roma, Italy;

${ }^{16}$ Fondazione IRCC Istituto Nazionale dei Tumori e Università di

Milano, Milano, Italy

${ }^{17}$ Fondazione IRCCS Cà Granda Ospedale Maggiore Policlinico,

Milano, Italy;

${ }^{18}$ Ospedale C e G Mazzoni, Ascoli Piceno, Italy;

${ }^{19}$ Ospedale Bianchi, Reggio Calabria, Italy;

${ }^{20}$ Ospedale Civile, Piacenza, Italy;

${ }^{21}$ Segreteria Nazionale GITMO, Genova, Italy and

${ }^{22}$ Italian Bone Marrow Donor Registry, Genova, Italy

E-mail: arambaldi@hpg23.it

\section{REFERENCES}

1 Greinix HT, Reiter E, Keil F, Fischer G, Lechner K, Dieckmann K et al. Leukemia-free survival and mortality in patients with refractory or relapsed acute leukemia given marrow transplants from sibling and unrelated donors. Bone Marrow Transplant 1998; 21: 673-678.

2 Michallet M, Thomas X, Vernant JP, Kuentz M, Socie G, Esperou-Bourdeau H et al. Long-term outcome after allogeneic hematopoietic stem cell transplantation for advanced stage acute myeloblastic leukemia: a retrospective study of 379 patients reported to the Societe Francaise de Greffe de Moelle (SFGM). Bone Marrow Transplant 2000; 26: 1157-1163.

3 Wong $R$, Shahjahan $M$, Wang $X$, Thall PF, De Lima $M$, Khouri I et al. Prognostic factors for outcomes of patients with refractory or relapsed acute myelogenous leukemia or myelodysplastic syndromes undergoing allogeneic progenitor cell transplantation. Biol Blood Marrow Transplant 2005; 11: 108-114.

4 Estey E. Treatment of refractory AML. Leukemia 1996; 10: 932-936.

5 Craddock C, Labopin M, Pillai S, Finke J, Bunjes D, Greinix H et al. Factors predicting outcome after unrelated donor stem cell transplantation in primary refractory acute myeloid leukaemia. Leukemia 2011; 25: 808-813.

6 Duval M, Klein JP, He W, Cahn J-Y, Cairo M, Camitta BM et al. Hematopoietic stem-cell transplantation for acute leukemia in relapse or primary induction failure. J Clin Oncol 2010; 28: 3730-3738.

7 Bacigalupo A, Ballen K, Rizzo D, Giralt S, Lazarus H, Ho V et al. Defining the intensity of conditioning regimens: working definitions. Biol Blood Marrow Transplant 2009; 15: 1628-1633.

8 Giralt S, Ballen K, Rizzo D, Bacigalupo A, Horowitz M, Pasquini M et al. Reduced-intensity conditioning regimen workshop: defining the dose spectrum. report of a workshop convened by the center for international blood and marrow transplant research. Biol Blood Marrow Transplant 2009; 15: 367-369.

9 Rambaldi A, Bacigalupo A, Fanin R, Ciceri F, Bonifazi F, Falda M et al. Outcome of patients activating an unrelated donor search: the impact of transplant with reduced intensity conditioning in a large cohort of consecutive high-risk patients. Leukemia 2012; 26: 1779-1785.

10 Mohty M, de Lavallade H, Ladaique P, Faucher C, Vey N, Coso D et al. The role of reduced intensity conditioning allogeneic stem cell transplantation in patients with acute myeloid leukemia: a donor vs no donor comparison. Leukemia 2005; 19: 916-920. 
11 Ciceri F, Bonini C, Stanghellini MT, Bondanza A, Traversari C, Salomoni M et al. Infusion of suicide-gene-engineered donor lymphocytes after family haploidentical haemopoietic stem-cell transplantation for leukaemia (the TK007 trial): a non-randomised phase I-II study. Lancet Oncol 2009; 10: 489-500.

12 lacobelli S. Suggestions on the use of statistical methodologies in studies of the European Group for Blood and Marrow Transplantation. Bone Marrow Transplant 2013; 48(Suppl 1): S1-S37.
13 Larson RA. Is secondary leukemia an independent poor prognostic factor in acute myeloid leukemia? Best Pract Res Clin Haematol 2007; 20: 29-37.

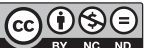

This work is licensed under a Creative Commons AttributionNonCommercial-NoDerivs 3.0 Unported License. To view a copy of this license, visit http://creativecommons.org/licenses/by-nc-nd/3.0/

Supplementary Information accompanies this paper on the Leukemia website (http://www.nature.com/leu)

\section{Clonal mast cell disease not meeting WHO criteria for diagnosis of mastocytosis: clinicopathologic features and comparison with indolent mastocytosis}

Leukemia (2013) 27, 2091-2094; doi:10.1038/leu.2013.227

Mastocytosis results from a clonal proliferation of morphological and immunophenotypically abnormal mast cells. ${ }^{1-4}$ Extracutaneous involvement is commonly seen in adults, and the diagnosis of systemic mastocytosis (SM) is established as per World Health Organization (WHO) criteria. ${ }^{5}$ Patients are frequently referred with episodic symptoms of 'mast cell activation' (MCA), wherein an underlying allergic or autoimmune disorder cannot be identified. ${ }^{6,7}$ In the absence of cutaneous involvement by mastocytosis, the next step typically is to determine whether SM is present. Increasingly, during such testing, patients who do not satisfy full diagnostic criteria for SM are identified; the major criterion (compact infiltrates of $\geqslant 15$ mast cells) is not met; instead, only one or two minor criteria are met. Previous reports have implicated such patients in the context of idiopathic anaphylaxis or anaphylaxis following Hymenoptera sting, ${ }^{8-10}$ however, the full spectrum of clinical presentation is currently not understood. Given the paucity of data regarding these patients, variously described as having monoclonal MCA syndrome (MMCAS) or pre-diagnostic indolent SM (ISM), ${ }^{11-13}$ we sought to describe their clinicopathologic characteristics, and to compare them with ISM cases, or those with MCA symptoms without clonal mast cells.

This study was approved by the Mayo Clinic Institutional Review Board and adhered to the tenets of the Declaration of Helsinki. We retrospectively studied consecutive patients who were referred to our institution for evaluation for SM. Every patient had symptoms attributed by the referring physician as being related to mast cell degranulation. The diagnostic assessment included a bone marrow biopsy with tryptase immunostaining and mast cell immunophenotyping for CD25/CD2 expression by flow cytometry and/or immunohistochemistry. Bone marrow histology was reviewed by two experienced hematopathologists (DC and $C A H$ ) with careful assessment for mast cell cytologic atypia (for example, spindling or hypogranularity), presence or absence of mast cell aggregates ( $\geqslant 15$ mast cells) and pattern of mast cell infiltration (that is, compact clusters versus singly distributed/interstitial). KITD816V analysis was performed using a sensitive $(0.01 \%)$ allelespecific PCR assay. ${ }^{7,14}$ The diagnosis and classification of SM was as per WHO criteria. ${ }^{5}$ After a full review of clinicopathological characteristics, three groups were identified: 'sub-diagnostic SM' (that is, those meeting 1-2 minor criteria for SM only), ISM (that is, those meeting $\mathrm{WHO}$ diagnostic criteria for SM) and those with MCA symptoms without clonal mast cells. In the group with subdiagnostic SM, those with cutaneous mast cell infiltration (for example, urticaria pigmentosa) were excluded. Similarly, patients with non-ISM were also excluded from the study. All statistical analyses considered clinical and laboratory parameters obtained at time of referral. Differences in the distribution of continuous variables between categories were analyzed by either MannWhitney or Kruskal-Wallis tests. Patient groups with nominal variables were compared by $\chi^{2}$ test. $P$-values $<0.05$ were considered significant. The Stat View statistical package (SAS Institute, Cary, NC, USA) was used for all calculations.

A total of 83 patients were studied; 40 patients had ISM, 21 had sub-diagnostic SM and 22 had MCA symptoms without clonal mast cells. Clinical and laboratory characteristics at the time of referral are shown (Table 1).

In the ISM group, 63\% exhibited cutaneous mast cell infiltration, $90 \%$ were KITD816V positive and $77 \%$ exhibited a baseline serum tryptase level $>20 \mathrm{ng} / \mathrm{ml}$ (Table 1). Thirty-three patients (83\%) exhibited multifocal compact mast cell infiltrates in bone marrow biopsy sections plus at least one minor criterion, whereas the remainder satisfied $\geqslant 3$ minor criteria.

In the sub-diagnostic SM group, none met the major SM diagnostic criterion (that is, compact mast cell infiltrates). Instead, the bone marrow mast cell infiltrate as identified by tryptase and in some cases CD117 immunostaining, was uniformly sparse with an interstitial distribution of individual mast cells (Figure 1). The estimated mast cell burden was $<5 \%$, and more typically $\leqslant 1 \%$ of total marrow cellularity. Twelve patients $(57 \%)$ demonstrated a population of overtly spindle-shaped mast cells and two patients (11\%) had a baseline serum tryptase level of $>20 \mathrm{ng} / \mathrm{ml}$ (Table 1). In terms of the WHO minor diagnostic criteria, 17 patients (81\%) with sub-diagnostic SM met only one criterion (KITD816V alone $=7$ or mast cell CD25/CD2 expression alone $=10)$. An additional four patients met two minor criteria (that is, KITD816V plus mast cell CD25/CD2 expression). Notably, three patients each did not undergo testing or had inconclusive results after screening for KITD816V or mast cell CD25/CD2 expression-here, even if the additional test were positive, criteria for SM would not have been met (that is, would have met $<3$ minor diagnostic criteria). A serum tryptase level above the normal range $(\geqslant 11.5 \mathrm{ng} / \mathrm{ml})$ and $>20 \mathrm{ng} / \mathrm{ml}$ was noted on at least one occasion during follow-up in $61 \%$ and $37 \%$ of sub-diagnostic 